\title{
Resonances, Chiral Symmetry, Coupled Channel Unitarity and Effective Lagrangians *
}

\author{
J. R. Peláez ${ }^{\mathrm{a}}$, J.A.Oller ${ }^{\mathrm{b}}$ and E.Oset ${ }^{\mathrm{c}}$ \\ a Departamento de Física Teórica. Universidad Complutense de Madrid \\ 28040 Madrid. SPAIN. \\ ${ }^{\mathrm{b}}$ Forschungzentrum Jülich, Institut für Kernphysik (Theorie) \\ D-52425 Jülich, GERMANY.
}

${ }^{\mathrm{c}}$ Departamento de Física Teórica and I.F.I.C. Centro Mixto Universidad de Valencia-C.S.I.C. 46100 Burjassot (Valencia), SPAIN.

\begin{abstract}
By means of a coupled channel non-perturbative unitary approach, it is possible to extend the strong constrains of Chiral Perturbation Theory to higher energies. In particular, it is possible to reproduce the lowest lying resonances in meson-meson scattering up to $1.2 \mathrm{GeV}$ using the parameters of the $O\left(p^{2}\right)$ and $O\left(p^{4}\right)$ Chiral Lagrangian. We report on an update of these results examining their possible relevance for meson spectroscopy.
\end{abstract}

\section{Introduction}

Chiral Perturbation Theory (ChPT) [1,2] has proved very successful in order to describe the physics of mesons at very low energies. The key point of the whole approach is to identify the lightest pseudoscalar mesons $\pi, K$ and $\eta$ as the Goldstone bosons associated to the chiral symmetry breaking. These particles will be the only degrees of freedom at low energies and their interactions can be described in terms of the most general effective Lagrangian which respects the chiral symmetry constraints.

So far as this is a low energy approach, the amplitude of a given process is basically given as an expansion in the external momenta over the scale of symmetry breaking $4 \pi v \simeq 1.2 \mathrm{GeV}$. It is also possible to calculate loops, which increase by two the order of the term in the chiral expansion and generate logarithmic contributions as well as divergences. The former are very important at low energies since they can dominate over some polynomial terms and the latter have to be absorbed in the renormalization of the free parameters that appear at the next order in the Lagrangian. It is therefore possible to obtain results which are finite to a given order in momenta. They provide a very good description of meson interactions up to about $500 \mathrm{MeV}$ in the best cases.

However, if one is interested in resonances in particular, as it happens in meson spec-

*Talk given at the 8th International Conference on Hadron Spectroscopy, HADRON99, August 24-28, 1999, Beijing, China. Work partially supported by DGICYT under contracts PB96-0753 and AEN97-1693 and by the EU TMR network eurodaphne contract no. ERBFMRX-CT98-0169. 
troscopy, it is little what one could do with just plain ChPT. In this work we will review recently proposed new nonperturbative schemes imposing unitarity to the chiral Lagrangian, thus enlarging the convergence of the chiral expansion and reproducing resonances. We will briefly comment on possible implications for meson spectroscopy.

\section{ChPT and Unitarity}

Within the coupled channel formalism, the unitarity of the $T$ matrix reads

$\operatorname{Im} T=T \operatorname{Im} G T^{*} \Rightarrow \operatorname{Im} T^{-1}=-\operatorname{Im} G \Rightarrow T=\left[\operatorname{Re} T^{-1}-i \operatorname{Im} G\right]^{-1}$,

where $\operatorname{Im} G$ is a known diagonal matrix, whose entries are just the phase space of the intermediate states. Indeed, $G$ is the integral of the propagators of the two particles in the intermediate state. Within ChPT the amplitudes are obtained as an expansion in powers of momenta, i.e. $T=T_{2}+T_{4}+\ldots$, where the subscript stands for the order in the expansion. Being basically a polynomial these amplitudes can only satisfy perturbative unitarity and they cannot yield poles and therefore resonances.

Within the Inverse Amplitude Method [3] [5], the ChPT expansion is only used for $\operatorname{Re} T^{-1}$, which is then used on the right hand side of eq.(11). This procedure ensures exact unitarity, while keeping the very same ChPT expansion at low energies. Using the complete $O\left(p^{4}\right)$ calculation, it was first applied to single channel $\pi \pi$ and $\pi K$ scattering and it was able to reproduce several isospin and angular momentum channels and to generate dynamically the $\sigma, \rho$ and $K^{*}$ resonances [3]. In principle, the IAM calculations up to order $n$ need the complete ChPT calculations up to the same order.

The next step was motivated by the results obtained when unitarizing the lowest order $O\left(p^{2}\right)$ ChPT scalar amplitudes using the Bethe-Salpeter equations (BS) [6]. Remarkably it was possible to fit the meson-meson scalar phase shifts up to $1.2 \mathrm{GeV}$ and reproduce the $\sigma, f_{0}$ and $a_{0}$ resonances, just by setting the cutoff to a natural value around $1 \mathrm{GeV}$, since there are no other free parameters. The link with the IAM was established in [ [I]: the BS solution is recovered from the IAM if one approximates $\operatorname{Re} T_{4} \simeq T_{2} \operatorname{Re} G T_{2}$.

But in order to obtain vector resonances the $O\left(p^{2}\right)$ is not enough. Unfortunately, the full $O\left(p^{4}\right)$ calculation is not available for all the meson-meson scattering channels. However it was also shown in [4] that in order to obtain a good results up to $1.2 \mathrm{GeV}$, it is enough to add to the BS approximation the $O\left(p^{4}\right)$ tree level, that is,

$$
\operatorname{Re} T_{4} \simeq \underbrace{T_{4}^{P}}_{O\left(p^{4}\right) \text { tree }}+\underbrace{T_{2} \operatorname{Re} G T_{2}}_{\text {s-channel loops }} .
$$

The results are remarkable, as it can be seen in Fig.1, where we display an updated fit to the meson-meson phase shifts and inelasticities [4,7]. Compared with our previous work [4], we have also corrected a small error in one amplitude, which only has a minor effect. It can be noticed that we are able to reproduce together the $\sigma, f_{0}, a_{0}, \rho, \kappa$ and $K^{*}$ resonances and the isospin zero state of the vector octet, $\omega_{8}$ ( see [4]). It is also possible to find, in the unphysical sheets, the poles associated to these resonances (see [四]).

In table I we list the values of the fitted IAM parameters. Note that within this approximation, tadpoles and crossed loops are neglected and absorbed in the chiral parameters, so that we cannot compare directly with the standard ChPT coefficients. Nevertheless 

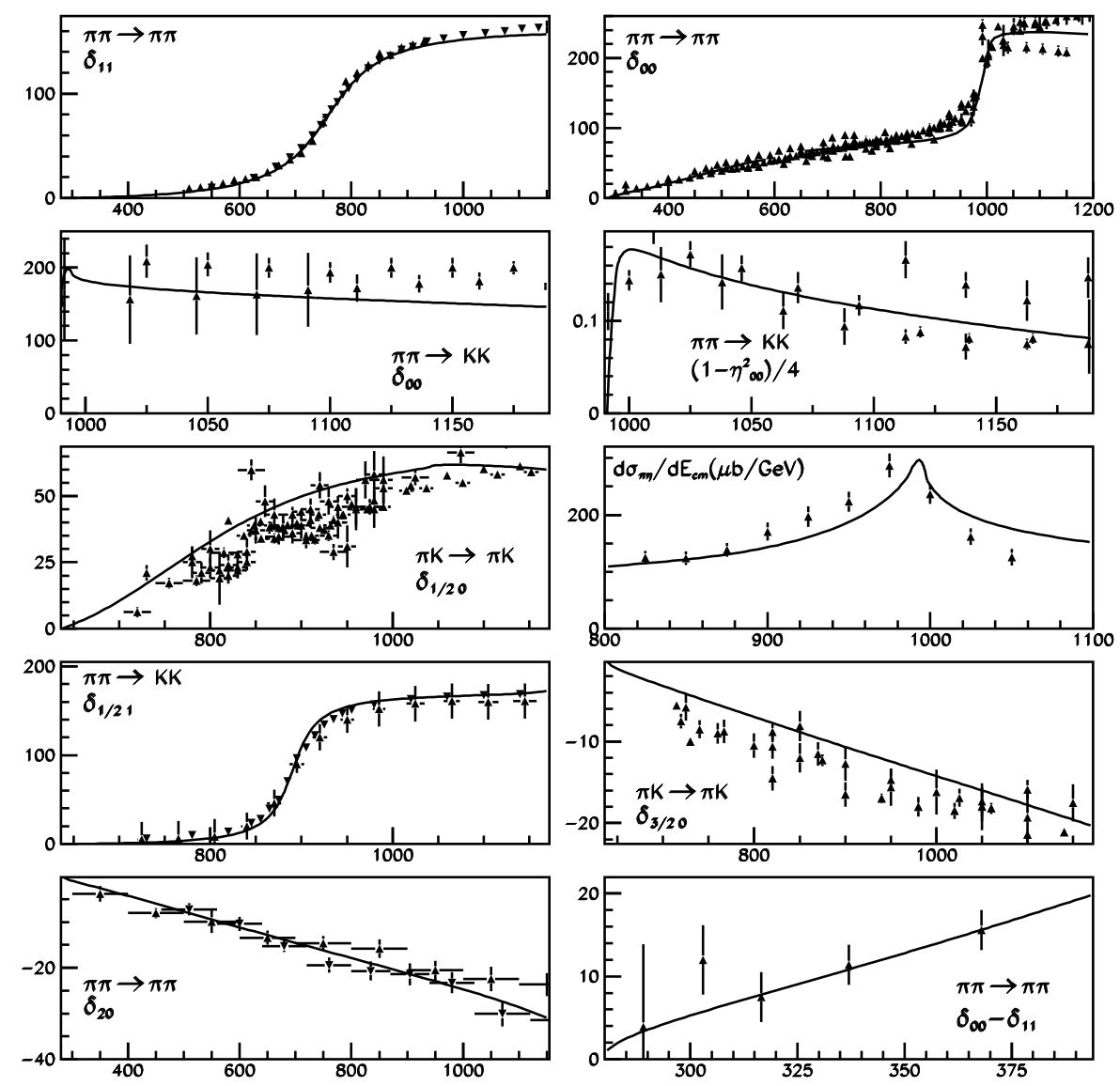

Figure 1. Meson-meson scattering results of the non-perturbative chiral approach. For the data see references in [3, [5,9].

they should be of the same order of magnitude, once the appropriate renormalization scale is used.

If we had the complete $O\left(p^{4}\right)$ ChPT calculation for all the meson-meson channels and we used the same renormalization scheme as in standard ChPT, the fitted parameters should be very similar to the standard ones. This has been checked with the single channel $\pi \pi$ and $\pi K$ scattering, but also by calculating the complete $2 \times 2 T$ amplitude for the $\pi \pi$, $K \bar{K}$ coupled channels [5]. In both cases the fitted parameters are perfectly compatible with those of standard ChPT. Further work is still in progress.

\section{Summary and Implications for Meson spectroscopy}

Chiral Perturbation Theory unitarized with the Inverse Amplitude Method, describes correctly the dynamics of meson-meson scattering in coupled channels, generating:

- The $\rho$ and the $K^{*}$, as well as a pole that corresponds to the $\omega_{8}$, which, all together, form the lightest vector octet. In order to obtain them it is necessary to include the $O\left(p^{4}\right)$ chiral parameters. The nonet is not reproduced since in the $\mathrm{SU}(3)$ limit the $\omega_{1}$ does not couple to two mesons. 
Table 1

Values of the chiral constants from the IAM fit and ChPT at their equivalent scales.

\begin{tabular}{|c|c|c|c|c|c|c|c|c|}
\hline & $L_{1}$ & $L_{2}$ & $L_{3}$ & $L_{4}$ & $L_{5}$ & $2 L_{6}+L_{8}$ & $L_{7}$ & $\mathrm{MeV}$ \\
\hline IAM & 0.88 & 1.54 & -3.66 & -0.27 & 1.09 & 0.68 & 0.10 & $q_{\max }=673$ \\
\hline ChPT & $0.4 \pm 0.3$ & $1.3 \pm 0.3$ & $-3.5 \pm 1.1$ & $-0.3 \pm 0.5$ & $1.3 \pm 0.5$ & $0.42 \pm 0.5$ & $-0.4 \pm 0.2$ & $\mu=800$ \\
\hline
\end{tabular}

- The $f_{0}, a_{0}, \sigma$ and $\kappa$. All their masses are below $1 \mathrm{GeV}$, and the last two are very wide, not Breit-Wigner resonances. All of them can be simply generated by unitarization of the lowest order ChPT, with just a cutoff as a free parameter.

The role of the $O\left(p^{4}\right)$ chiral parameters can be understood 2, 8] by writing a Lagrangian with pions, kaons and etas, but also with heavier resonances coupled in a chirally invariant way. Then one integrates out these heavier states and the resulting Lagrangian is that of ChPT, but now the values of the chiral constants can be related to the masses and widths of the preexisting heavier resonances ("Resonance Saturation Hypothesis"). Most of the experimental values of the chiral coefficients are saturated by these estimates due to vector resonances alone (that is vector meson dominance) but some other parameters still need the existence of scalar states. Recently [9], using the N/D unitarization method with explicit resonances added to the lowest order ChPT Lagrangian, it has been established that these heavier scalar states should appear with a mass around $1.3-1.4 \mathrm{GeV}$ for the octet and $1 \mathrm{GeV}$ for the singlet. In addition, the $\sigma, \kappa, a_{0}$ and a strong contribution to the $f_{0}$, were also generated from the unitarization of the ChPT lowest order. These states still survive when the heavier scalars are removed. That agrees with our observation that the $\sigma, \kappa, f_{0}$ and $a_{0}$ are generated independently of the chiral parameters, that is, of the preexisting scalar nonet, which is heavier.

Since Chiral Perturbation Theory does not deal with quarks and gluons, it is very hard to make any conclusive statement about the nature of these states $(q \bar{q}$, four-quark, molecule, etc...), unless we make additional assumptions. However, it seems clear that the nature of the lightest scalar mesons is different from that of vectors. In addition, the fact that we obtain simultaneously the above nine scalar resonances with the same procedure, seems to indicate that they are good candidates to form a complete $S U(3)$ nonet.

\section{REFERENCES}

1. $\quad$ S. Weinberg, Physica A96 (1979) 327.

2. J Gasser annd H. Leutwyler, Ann. Phys. NY 158 (1984) 142; J. Gasser and H. Leutwyler, Nucl. Phys. B250 (1985) 465,517,539.

3. T. N. Truong, Phys. Rev. Lett. 661 (1988) 2526; Phys. Rev. Lett. 67 (1991) 2260; A. Dobado, M.J.Herrero and T.N. Truong, Phys. Lett. B235 (1990) 134 ; A. Dobado and J.R. Peláez,Phys. Rev. D47 4883 (1993); Phys. Rev. D56 (1997) 3057.

4. J.A. Oller, E. Oset and J.R. Peláez, Phys. Rev. Lett. 80 (1998) 3452; Phys. Rev. D59 (1999) 074001.

5. F. Guerrero and J. A. Oller, Nucl. Phys. B537 (1999) 459.

6. J.A. Oller and E. Oset, Nucl. Phys. A620 (1997) 438; erratum Nucl. Phys. A624 (1999) 407.

7. J.A. Oller, E. Oset and J.R. Peláez in preparation.

8. G. Ecker, J. Gasser, A. Pich and E.de Rafael, Nucl. Phys. B321 (1989) 311.

9. J.A. Oller and E. Oset, Phys. Rev. D60, 074023 (1999). 